\title{
Relação entre a competência funcional da memória episódica e os fatores associados à independência funcional de idosos saudáveis
}

\author{
Relationship between the functional competence of episodic memory and the factors associated \\ with functional independence of healthy elderly
}

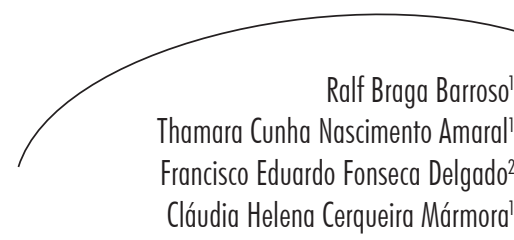

Resumo

Objetivo: O objetivo deste estudo foi descrever a relação entre competência funcional da memória episódica e fatores associados à independência de idosos saudáveis do município de Juiz de Fora, Minas Gerais, Brasil. Métodos: Trata-se de estudo transversal, descritivo e exploratório em que 60 idosos saudáveis compuseram a amostra final. Para avaliar a capacidade funcional, foram utilizadas as escalas de Katz e de Lawton \& Brody. A memória episódica foi avaliada utilizando-se teste de West \& Thorn, validado e adaptado para a população brasileira por Yassuda, Lasca e Neri em 2005. A análise estatística foi realizada por meio do software estatístico Sphinx Léxica e Eureka, versão 5.0. Resultados: Foi encontrada associação estatisticamente significante entre escolaridade e desempenho em tarefa de memória episódica e entre esta e três itens da escala de avaliação das atividades instrumentais de vida diária, embora inconclusiva. Conclusões: Acredita-se que esses resultados se devam ao fato de existir muitas diferenças

Palavras-chave: Fisioterapia. Memória Episódica. Envelhecimento. Idoso. Dependência. entre os estudos com relação à metodologia empregada e a escolha dos instrumentos que avaliam tanto a memória episódica, quanto a capacidade funcional de idosos. Além disso, a hipótese de que no envelhecimento saudável existe uma capacidade de, frente às perdas graduais na memória episódica, compensá-las utilizando outras estratégias e recursos, deve receber atenção de futuras pesquisas.

\section{Abstract}

Objective: This study aimed to describe the relationship between the functional competence of episodic memory and its associations with functional independence of healthy elderly in the city of Juiz de Fora, Minas Gerais state, Brazil. Methods: It is a cross-sectional, descriptive and exploratory research in which 60 healthy elderly comprised the final sample. To evaluate the functional capacity, the Index of

\footnotetext{
Departamento de Fisioterapia do Idoso, do Adulto e Materno-infantil, Faculdade de Fisioterapia. Universidade Federal de Juiz de Fora. Juiz de Fora, MG, Brasil.

2 Curso de Fisioterapia. Universidade Presidente Antônio Carlos - Campus II. Juiz de Fora, MG, Brasil. 
Independence of Daily Living and the Lawton Instrumental Activities of Daily Living Scale were used. The episodic memory was assessed by a test of West \& Thorn, adapted and validated for the Brazilian population by Yassuda, Lasca e Neri in 2005. Statistical analysis were performed using the 5.0 version of the statistical software Sphinx Léxica and Eureka. Results: Statistically significant association between education level and performance in episodic memory task was found, as well as between the latter and three items of assessment scale of instrumental activities of daily living, although inconclusive. Conclusions: It is believed that these results are due to the fact that there are so many differences between researches with respect to methodology and the choice of instruments that evaluates both episodic memory and the erderly's functional capacity. Furthermore, the hypothesis that in healthy aging there is a capacity to face gradual losses in episodic memory and compensate them using other strategies and resources, should receive attention in further research.
Key words: Physical Therapy Specialty. Memory, Episodic. Aging. Elderly. Dependency.

\section{INTRODUÇÃO}

A queixa de perda de memória é muito frequente entre idosos, ${ }^{1}$ havendo grande concordância de que isso é um fenômeno do envelhecimento normal do ser humano. Por ser uma queixa frequente na terceira idade, o declínio observado na memória com o envelhecimento tem sido foco de diversos estudos ${ }^{1-5}$ que visam entender de que forma esse processo afeta a vida do geronte.

A memória divide-se em dois grandes grupos: a memória declarativa ou explícita e a memória não declarativa ou implícita. A primeira se subdivide ainda em memória episódica (fatos e eventos) e semântica (materiais linguísticos). Memória associativa e de procedimentos são subtipos da memória implícita, expressas por meio de respostas fisiológicas ou mudanças de comportamento, sem acesso consciente. ${ }^{6}$ Kinm \& Giovanello ${ }^{2}$ definem a memória episódica como uma memória de eventos e experiências que acontecem no passado de um indivíduo.

Salthouse $^{3}$ descreve que o declínio nas habilidades mnemônicas está relacionado à teoria do desuso, em que o estilo de vida assumido pelo idoso pode influenciar de forma negativa o funcionamento da memória. Durante o processo de envelhecimento, são frequentemente adotadas condutas que levam os indivíduos a permanecerem em uma situação que oferece poucos estímulos cognitivos, prejudicando a aquisição, consolidação e evocação de um fato ou evento. Portanto, esse desuso acaba por gerar perda da capacidade cognitiva, além da perda neuronal e sináptica inerente ao envelhecer.

Nota-se que, no processo de envelhecimento, as perdas relativas à memória episódica são maiores do que as relativas à memória semântica. Sabe-se também que, no envelhecimento saudável, a memória implícita é a última a ser afetada. ${ }^{4}$ Dessa forma, torna-se importante investigar os fatores associados a esta perda, como forma de compreender sua relação com a qualidade de vida e a independência dos idosos para realizar suas atividades rotineiras.

Apesar de os déficits de memória serem parte do envelhecimento saudável, de forma controversa, seu bom funcionamento é essencial para a capacidade funcional do idoso. ${ }^{5} \mathrm{~A}$ capacidade funcional é um dos importantes marcadores de um envelhecimento bem-sucedido e da qualidade de vida de idosos. ${ }^{7}$ Vale salientar também a diferença entre "desempenho" e "capacidade funcional". O primeiro avalia o que o idoso realmente faz no seu dia a dia; o segundo, por sua vez, avalia o potencial que o indivíduo possui para realizar a atividade - isto é, sua capacidade remanescente, que pode ou não ser utilizada. ${ }^{8}$ 
Vários autores encontraram correlação entre idade avançada, ${ }^{9-15}$ baixa escolaridade, ${ }^{9,10,12,14-17}$ pior condição socioeconômica, ${ }^{9,12,13}$ déficit $\operatorname{cognitivo~}^{14,16}$ e sexo feminino ${ }^{9,11,13}$ com a perda de funcionalidade no idoso.

A capacidade funcional é mensurada por meio de instrumentos padronizados que avaliam o desempenho do idoso para realização das atividades básicas de vida diária (ABVD), como tomar banho, vestir-se, ir ao banheiro, e atividades instrumentais de vida diária (AIVD), como telefonar, fazer compras e cuidar das finanças. ${ }^{18}$ Paixão-Junior \& Reichenrein ${ }^{19}$ não encontraram nenhum instrumento com a finalidade de avaliação da independência dos idosos para as atividades avançadas de vida diária (atividades voluntárias sociais, ocupacionais e de recreação).

Para Ramos et al., ${ }^{20}$ o comprometimento cognitivo associado à dependência nas atividades do dia a dia está fortemente relacionado com mortalidade em idosos residentes em centros urbanos no Brasil.

Todas as questões levantadas proclamam a necessidade de se ampliar a compreensão sobre as variáveis que estão relacionadas aos processos mnemônicos, visto que a funcionalidade do idoso está relacionada à sua saúde e sua qualidade de vida, e são relevantes para as decisões clínicas no contexto do envelhecimento. ${ }^{21}$

$\mathrm{Na}$ literatura internacional, observaramse alguns estudos em que se buscou verificar a existência de correlação entre memória episódica, funções executivas e independência nas atividades de vida diária. ${ }^{22-24}$ Tucker-Drob ${ }^{22}$ afirma que essa correlação deve ser mais bem elucidada, uma vez que os critérios diagnósticos de demência estão baseados no déficit de memória associado à perda de independência funcional. Isso poderia sugerir que o que diferencia o envelhecimento saudável do patológico seria justamente uma perda de memória não acompanhada de uma perda da capacidade funcional. No entanto, os estudos supracitados ${ }^{22-24}$ apresentam resultados controversos e, no Brasil, tais pesquisas são escassas. Investigar essa associação no contexto brasileiro pode contribuir para melhor compreensão da interação da memória episódica com a execução das atividades de vida diária em idosos, bem como destas com outras variáveis, levantando questões que servirão de subsídios para pesquisas futuras. Isto permite planejar condutas e políticas públicas eficazes, de forma a promover qualidade de vida e melhorar aspectos biopsicossociais desses idosos.

Sendo assim, o objetivo deste estudo foi descrever a relação entre a competência funcional da memória episódica e os fatores associados à independência de idosos saudáveis do município de Juiz de Fora, Minas Gerais, Brasil.

\section{MÉTODOS}

Trata-se de estudo transversal, descritivo e exploratório, em que foram recrutados 64 idosos, aleatoriamente, de centros de convivência para a terceira idade da cidade Juiz de Fora-MG, do Serviço de Fisioterapia do Hospital Universitário da Universidade Federal Juiz de Fora (HU/UFJF) e também idosos abordados na comunidade diretamente pelos pesquisadores do presente estudo.

Como critérios de inclusão, foram estabelecidos: ter 60 anos de idade ou mais, ser alfabetizado, não possuir diagnóstico de doenças incapacitantes, demência, depressão e doenças crônicas controladas. Esses dados foram obtidos de forma direta durante a abordagem inicial aos sujeitos e confirmados por meio do questionário semiestruturado aplicado posteriormente. Os critérios de exclusão foram: dificuldade de compreensão na execução das tarefas propostas e dificuldade de leitura e identificação, no momento da realização dos procedimentos, de algum dos critérios não compatíveis com os de inclusão do presente estudo.

Todos os instrumentos foram inicialmente aplicados em uma sala, individualmente, com cada idoso, nas dependências da Faculdade de 
Fisioterapia da UFJF. Em seguida, foi cedida uma sala em um polo para a terceira idade pertencente à mesma instituição, onde todos os instrumentos passaram a ser aplicados da mesma forma. Além disso, quando o idoso apresentasse alguma dificuldade de deslocamento para os lugares predeterminados, o estudo poderia ser desenvolvido em sua residência, desde que esta oferecesse um local com privacidade para o idoso reportar as informações aos pesquisadores, luminosidade adequada e não houvesse fatores que poderiam desviar sua atenção. $\mathrm{O}$ processo de coleta de dados foi realizado nos períodos da manhã e tarde, entre janeiro e julho de 2012.

Após as coletas, quatro dos 64 sujeitos foram excluídos: um por ter relatado uso de antidepressivos, outro por possuir hipótese diagnóstica de demência de Alzheimer, o terceiro por exibir dificuldades de leitura e o último pelo fato de o local eleito por ele, para aplicação dos questionários, escalas e testes, não ter oferecido as condições descritas anteriormente. Portanto, a amostra final foi composta por 60 idosos.

Os idosos aptos a participar da pesquisa assinaram o Termo de Consentimento Livre e Esclarecido (TCLE), e posteriormente foi aplicado um questionário semiestruturado contendo dados pessoais e hábitos de vida, como nome, idade, estado civil, situação trabalhista, doenças atuais ou pregressas, escolaridade, moradia, medicamentos em uso, atividade física (frequência e intensidade), atividades de lazer, entre outras.

As escalas de $\mathrm{Katz}^{25}$ e Lawton \& Brody ${ }^{26}$ foram os instrumentos eleitos para a avaliação da independência dos idosos quanto a ABVDs e AIVDs, respectivamente. A escala de Katz ${ }^{25}$ encontra-se validada para a população brasileira. ${ }^{18}$ Não foi encontrado nenhum estudo de validação da escala de Lawton \& Brody, ${ }^{26}$ mas PaixãoJunior \& Reichenrein ${ }^{19}$ encontraram 23 estudos brasileiros que a utilizaram como instrumento, mostrando que essa escala é amplamente adotada no país.
$\mathrm{Na}$ escala de Katz, são realizadas perguntas com respostas dicotômicas (sim ou não), onde cada resposta afirmativa equivale a um ponto. $\mathrm{Na}$ somatória final, os idosos com pontuação igual a 5 ou 6 são classificados como independentes; aqueles com 4 ou 3 pontos são classificados como parcialmente dependentes; e os com 2 pontos ou menos, totalmente dependentes. ${ }^{25}$ Para a escala de Lawton \& Brody, cada questão possui três alternativas, com seus respectivos valores: realiza sem ajuda (três pontos); realiza com ajuda parcial (dois pontos); e não consegue realizar (um ponto). A pontuação máxima que pode ser obtida é de 27 pontos. Sendo assim, são considerados independentes aqueles com pontuação entre 19 e 27; dependentes parciais com pontuação entre 10 e 18 pontos; e dependentes, aqueles com pontuação abaixo de nove pontos. ${ }^{26}$

Após a aplicação dessas escalas, foi aplicado o teste de memória episódica, que consiste de quatro listas de supermercado escritas por West \& Thorn,${ }^{27}$ validadas e adaptadas ao português por Yassuda et al. ${ }^{4}$ As autoras validaram e adaptaram essas listas com a finalidade de obter um instrumento capaz de avaliar inicialmente o desempenho em memória episódica, realizar uma intervenção, e ter outras listas equivalentes em grau de dificuldade que pudessem ser usadas como forma de reavaliação deste desempenho após as intervenções. Como se trata de estudo transversal em que se realiza uma única avaliação, resolveu-se aplicar somente duas listas (lista A e lista B), com um intervalo de aproximadamente um minuto entre elas, sendo considerado o melhor resultado pelos pesquisadores.

O motivo desta conduta se baseia no fato de que, após a aplicação de testes de memorização consecutivos, há o chamado "processo de interferência proativa", em que a memorização dos itens de uma primeira lista interfere na evocação dos itens de uma segunda. ${ }^{28}$ No entanto, resolveu-se aplicar mais uma lista, caso o idoso não tivesse entendido completamente a tarefa proposta, para se ter mais uma chance de avaliar seu real desempenho no teste de memória 
episódica proposto e verificar se existiu efeito proveniente do aprendizado e aperfeiçoamento da tarefa. Os idosos tiveram cinco minutos para memorizar os itens contidos em cada lista e, posteriormente, três minutos para escrever o que fosse lembrado de cada uma delas, tendo sido pontuados por cada item lembrado.

Todos os questionários, escalas e testes foram aplicados por dois acadêmicos treinados e que realizaram previamente estudo-piloto com alguns idosos para aumentar a confiabilidade interexaminador.

A análise estatística dos dados foi realizada por meio do software estatístico Sphinx Lexica \& Eureka, versão5.0. Na primeira etapa, foi realizado o teste de Levine para verificar a normalidade dos dados submetidos à análise para $(\mathrm{p}<0,05)$. Após constatação de que os dados se distribuíram de forma aproximadamente normal, foram realizados procedimentos de análise estatística descritiva, por meio da plotagem de gráficos de barras, que são utilizados quando se tem uma escala numérica comum. Os dados dos valores assim obtidos tiveram como objetivo demonstrar o comportamento das variáveis "Independência para as atividades de vida diária". $\mathrm{Na}$ segunda etapa da análise estatística, todas as variáveis foram organizadas de forma categórica nominal e utilizou-se o teste qui-quadrado para verificar a existência de associações ou dissociações entre elas, sendo considerado, para tal, o nível de significância de $(p<0,05)$. Nesta etapa, os resultados dos testes de independência funcional foram analisados de duas formas diferentes: uma considerando somente os escores totais de cada teste; e a outra, considerando cada item das escalas das atividades básicas e instrumentais de vida diária. Posteriormente, foram inseridas somente as variáveis numéricas, e para verificar a existência de correlação entre elas, utilizouse o coeficiente de correlação de Pearson, para $(\mathrm{p}<0,05)$.

Este estudo cumpriu os princípios éticos contidos na Declaração de Helsinki e foi aprovado pelo Comitê de Ética em Pesquisa da Universidade Federal de Juiz de Fora-CEP/UFJF, sob o parecer $n^{\circ} 018 / 2011$, além do atendimento à legislação pertinente.

\section{RESULTADOS}

A média de idade dos 60 idosos foi 69,55 anos (dp $\pm 7,72)$ com coeficiente de variação de $11 \% ; 51,7 \%$ dos idosos tinham até 68 anos, revelando ainda predominância de idosos com idades mais baixas. Quanto ao sexo, 90\% eram do sexo feminino. A média de anos de estudos completados apresentada pela amostra foi de 9,67 anos (dp $\pm 4,86)$, com coeficiente de variação de $50,2 \%$. A análise revelou que $36,7 \%$ dos sujeitos tinham 12 anos ou mais de escolaridade. Quanto à situação trabalhista, 88,3\% eram aposentados, pensionistas ou nunca trabalharam. Em relação às idosas, das 49 inativas, 93,9\% eram aposentadas e $6,1 \%$ eram somente pensionistas. Quase metade $(41,7 \%)$ dos idosos possuía uma renda mensal de um a dois salários mínimos. A média de desempenho em tarefa de memória episódica foi 14,05 itens (dp $\pm 6,43)$, com coeficiente de variação de $45,8 \%$, sendo que $41,7 \%$ dos idosos se lembraram de sete a 13 itens em uma das listas. A figura 1 apresenta estes achados de forma mais minuciosa e a distribuição por frequências desse desempenho. 


\begin{tabular}{|l|r|r|}
\hline 10 pontos & 4 & $\mathbf{6 , 7 \%}$ \\
\hline 11 pontos & 4 & $6,7 \%$ \\
\hline 12 pontos & 4 & $6,7 \%$ \\
\hline 13 pontos & 2 & $3,3 \%$ \\
\hline 14 pontos & 3 & $5,0 \%$ \\
\hline 15 pontos & 9 & $15,0 \%$ \\
\hline 17 pontos & 1 & $1,7 \%$ \\
\hline 19 pontos & 5 & $8,3 \%$ \\
\hline 20 pontos & 1 & $1,7 \%$ \\
\hline 22 pontos & 1 & $1,7 \%$ \\
\hline 23 pontos & 1 & $1,7 \%$ \\
\hline 24 pontos & 1 & $1,7 \%$ \\
\hline 25 pontos & 2 & $3,3 \%$ \\
\hline 26 pontos & 3 & $5,0 \%$ \\
\hline 27 pontos & 1 & $1,7 \%$ \\
\hline 5 pontos & 2 & $3,3 \%$ \\
\hline 6 pontos & 3 & $5,0 \%$ \\
\hline 7 pontos & 2 & $3,3 \%$ \\
\hline 8 pontos & 3 & $5,0 \%$ \\
\hline 9 pontos & 6 & $10,0 \%$ \\
\hline$\ldots$ & 2 & $3,3 \%$ \\
\hline Total & 60 & $100,0 \%$ \\
\hline
\end{tabular}

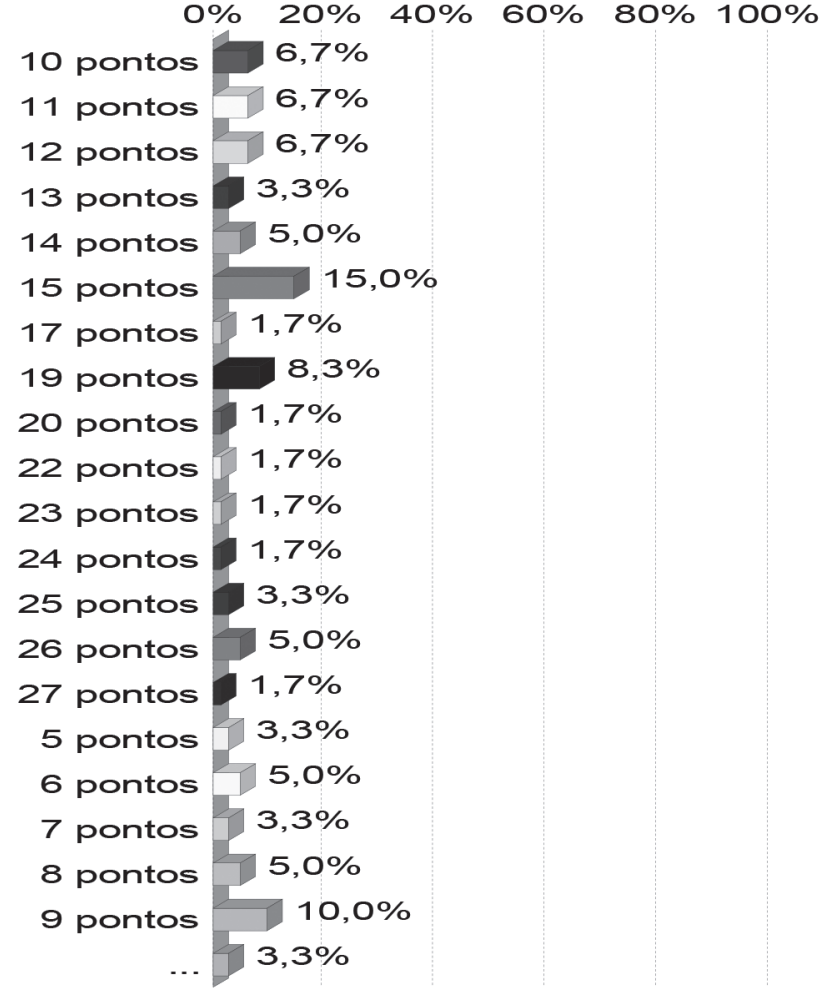

Figura 1. Frequências de desempenho em memória episódica. Juiz de Fora-MG, 2012.

Dentre os voluntários, $70 \%$ dos idosos relataram praticar algum tipo de atividade física regular e 63,3\% relataram realizar alguma outra atividade de lazer. Ao serem questionados sobre dificuldades para enxergar, 97,6\% responderam que sim; $23,3 \%$ dos idosos relataram também dificuldades em ouvir; e $70 \%$ responderam que possuíam esquecimentos frequentes.

Nosinstrumentos que avaliamaindependência funcional do idoso, todos os participantes foram classificados como totalmente independentes. No entanto, algumas funções apresentaram declínio. Na escala de Katz, ${ }^{25}$ 10\% dos voluntários obtiveram pontuação igual a 5 e os outros $90 \%$ apresentaram escore máximo (6 pontos). $\mathrm{Na}$ escala de Lawton \& Brody, ${ }^{26}$ 1,7\% dos idosos obtiveram pontuação inferior a $21,5 \%$ entre $21 \mathrm{e}$ 23 pontos, e $93,3 \%$ obtiveram 23 ou mais pontos, sendo que, destes, 76,8\% relataram ser capazes de realizar todas as atividades desta escala sem nenhuma ajuda. $\mathrm{Na}$ avaliação das atividades instrumentais de vida diária, a atividade em que os idosos apresentaram maior dependência foi "conseguir realizar pequenos trabalhos manuais, como pequenos reparos". Nas atividades básicas de vida diária, a única que apresentou declínio foi a continência. Observou-se também maior declínio nas atividades instrumentais do que nas atividades básicas na amostra estudada. As figuras 2 e 3 evidenciam este comportamento e mostram a independência dos idosos por atividades em cada escala. 


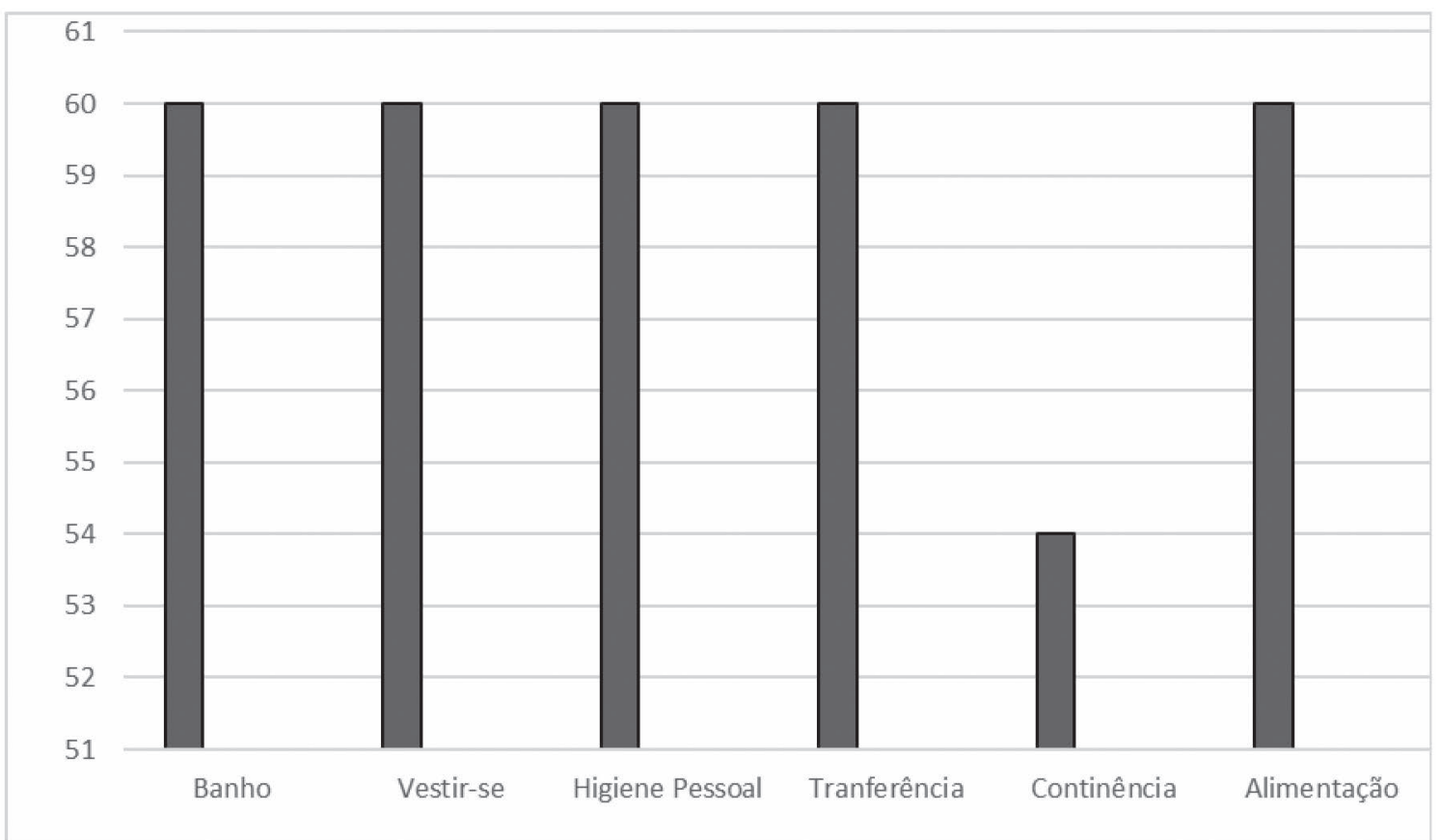

Figura 2. Independência para as ABVDs. Juiz de Fora-MG, 2012.

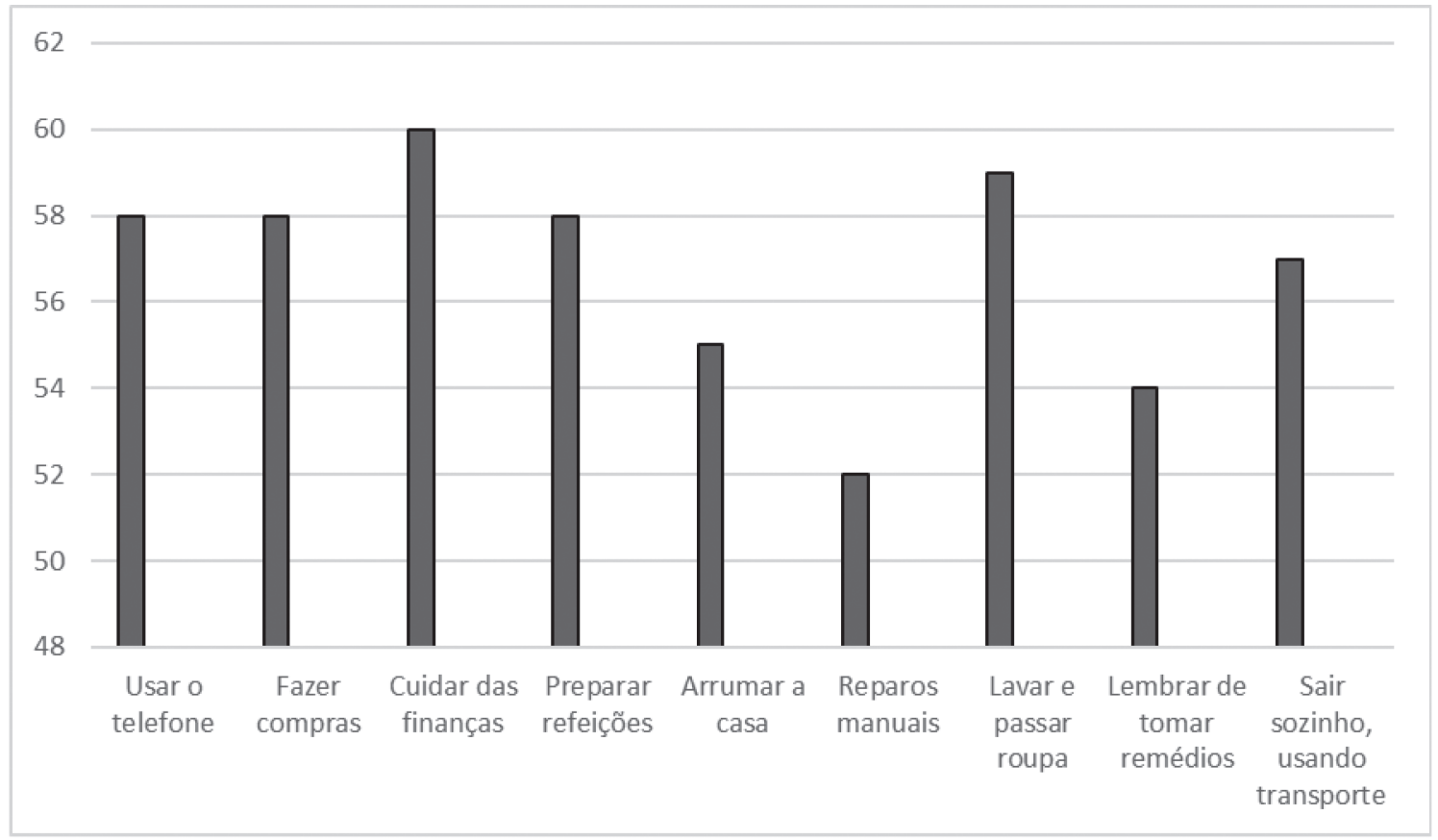

Figura 3. Independência para as AIVDs. Juiz de Fora-MG, 2012. 
Ao aplicar o teste qui-quadrado, as variáveis "prática de atividade física" e "prática de atividade de lazer" revelaram associação estatisticamente significativa $(\mathrm{p}<0,05)$. Quando foram analisados o desempenho em tarefa de memória episódica com cada item das atividades de vida diária, três itens das atividades instrumentais (arrumar a casa, fazer compras e sair de casa sozinho para locais distantes, utilizando transporte público não adaptado) associaram-se, com significância estatística, com o desempenho em memória episódica $(\mathrm{p}<0,05)$. No entanto, a análise com os escores totais das escalas de $\mathrm{Katz}^{25}$ e Lawton \& Brody $^{26}$ não esteve associada à tarefa de memória episódica.

Com o coeficiente de correlação de Pearson, somente escolaridade e desempenho no teste de memória episódica apresentaram correlação com significância estatística $(r=0,64 ; p<0,05)$.

\section{DISCUSSÃO}

Segundo dados do Censo 2010, ${ }^{29}$ aproximadamente $55,1 \%$ dos idosos brasileiros têm entre 60 e 69 anos, justificando a alta predominância de idosos jovens na amostra. Dados do mesmo Censo ${ }^{29}$ mostram também que dividindo-se idosos por faixas etárias e sexo, o número de mulheres é sempre maior que o de homens, e que em faixas etárias mais avançadas, o número de idosas chega a ser quase o dobro do de idosos.

Quanto ao desempenho em tarefa de memória episódica, foram utilizadas duas listas (A e B) validadas e adaptadas transculturalmente por Yassuda et al., ${ }^{4}$ considerando-se o melhor resultado. A média de itens lembrados pela amostra do presente estudo foi de 14,05, sendo similar a das quatro listas do estudo citado. ${ }^{4}$ A média de anos estudados pelos idosos da pesquisa das referidas autoras ${ }^{4}$ foi de 10,4 e a de idade foi de 62,6 anos. Neste estudo, a média de idade dos idosos foi maior e a de anos completos estudados foi menor.
Embora não encontrado neste trabalho, o declínio de memória episódica associado ao aumento da idade é bem reportado na literatura. ${ }^{30-33}$ Acredita-se que esses resultados possam ser justificados pela menor idade dos idosos estudados. De acordo com Small et al., ${ }^{33}$ existe um declínio leve na memória episódica antes dos 75 anos de idade, mas este se acentua somente após essa idade. Além disso, acreditase que se houvesse um grupo controle de jovens para confrontar com o desempenho dos idosos, tal diferença seria evidenciada, mas isso não fez parte dos objetivos deste estudo.

Os resultados apresentados demonstraram que existe associação entre escolaridade e desempenho em memória episódica. Tal achado é corroborado pela literatura, em que, segundo alguns autores, a educação desempenha papel fundamental para uma memória bem-sucedida no envelhecimento. ${ }^{32-34}$ Zahodne et al. ${ }^{32}$ descreveram um melhor desempenho em memória episódica de idosos com alta escolaridade, comparada com idosos de baixa escolaridade e mesma idade. No entanto, o declínio observado na memória episódica ao longo dos anos foi semelhante em ambos os grupos, sugerindo que a escolaridade pouco influencia no curso da memória no processo de envelhecimento.

Quanto às variáveis sociodemográficas e capacidade funcional, o presente estudo não encontrou nenhuma associação entre esta e aquelas, embora sejam bem documentadas na literatura. ${ }^{9-16}$ Siqueira et al. ${ }^{17}$ também não encontraram associação entre funcionalidade e idade em idosos internados. Os autores justificaram esse achado pela baixa capacidade funcional dos idosos no início da internação, o que não foi corroborado pelo presente estudo, em que todos os idosos foram classificados como independentes tanto para as atividades básicas, quanto para as instrumentais de vida diária. Outros estudos também não evidenciaram da mesma forma tal associação. ${ }^{11,15,24}$ Farias et al. ${ }^{23}$ não encontraram relação entre idade, escolaridade, sexo e independência em atividades instrumentais de vida diária. Acredita-se que a 
falta de padronização dos instrumentos utilizados para avaliação da capacidade funcional entre os estudos, assim como os critérios de seleção da amostra estudada, possam justificar, ao menos em parte, as divergências entre esses resultados.

Embora não tenha sido objetivo do presente estudo verificar a prevalência de incapacidade funcional na população representada, por se tratar de amostra de conveniência composta, em sua maioria, por idosos de centros de convivência, com perfil mais ativo e engajado socialmente, vale citar que todos os indivíduos foram classificados como independentes tanto pela escala de Katz, ${ }^{25}$ quanto pela de Lawton \& Brody. ${ }^{26}$ Maciel \& Guerra ${ }^{16}$ encontraram, por sua vez, prevalência de incapacidade funcional de $13,2 \%$ pela escala de Katz e de 52,6\% na escala de Lawton \& Brody na amostra estudada.

Ao confrontar os gráficos de independência dos idosos para as ABVDs com o de AIVDs, observa-se que esta declina primeiro que aquela no processo de envelhecimento bem-sucedido. Maciel \& Guerra ${ }^{16}$ descrevem que as ABVDs são atividades elementares, inerentes à sobrevivência do indivíduo; por esse motivo, espera-se que seu declínio ocorra somente após o declínio nas AIVDs, que são atividades mais complexas.

No presente trabalho não foi encontrada nenhuma associação entre desempenho em memória episódica com as escalas de $\mathrm{Katz}^{25}$ e Lawton \& Brody, ${ }^{26}$ sugerindo não haver correlação entre memória episódica e capacidade funcional no processo de envelhecimento saudável, quando na análise foram utilizados escores totais de cada escala. Tucker-Drob ${ }^{22}$ afirma que essa questão constitui um paradoxo. $O$ autor argumenta de forma crucial que a diretriz para exclusão do diagnóstico de demência em adultos, como sendo pessoas que têm déficit cognitivo sem declínio funcional, acaba por deixar implícita a perspectiva de que o envelhecimento saudável resulta, frequentemente, em declínio cognitivo não acompanhado de prejuízo funcional.

Tucker-Drob ${ }^{22}$ expõe também que, embora as tarefas diárias sejam dependentes do processamento cognitivo, a quantidade deste processamento requerida para executar tais tarefas é tão pequena que o desempenho funcional só será afetado por formas graves de declínios na cognição associada a quadros demenciais. Vale ressaltar que o estudo desse autor ${ }^{22}$ apresenta dois resultados muito interessantes. Primeiro, o autor encontrou forte correlação entre alterações cognitivas, incluindo a memória episódica como variável, e mudanças na execução das atividades de vida diária, quando estas foram avaliadas de forma objetiva por um dos pesquisadores do estudo. No entanto, o segundo resultado demonstrou que quando a capacidade de realização das atividades de vida diária foi mensurada pelo autorrelato dos idosos, não houve associação entre cognição e funções da vida diária. Além disso, essas funções mensuradas objetivamente e autorreportadas tiveram fraca correlação, indicando que os idosos não são bons juízes sobre sua capacidade funcional. ${ }^{22}$ Estes achados são de suma importância, pois muitos clínicos e muitas pesquisas, como no caso desta, utilizam instrumentos em que o idoso relata sua capacidade de executar as tarefas diárias, o que pode não constituir, na maioria das vezes, uma avaliação fidedigna.

Farias et al. ${ }^{23}$ encontraram associação significante entre memória episódica e independência nas atividades instrumentais de vida diária em estudo longitudinal de cinco anos. Cahn-Weiner et al. ${ }^{24}$ também encontraram a mesma associação, embora tenham demonstrado que somente funções executivas, que não foram avaliadas pelo presente estudo, foram a variável que previu independentemente declínio ao longo do tempo nas AIVDs.

Um aspecto merece destaque em relação a esses dois trabalhos: ambos os estudos incluíram em suas amostras indivíduos com comprometimento cognitivo leve e demência de Alzheimer, diferentemente deste, que incluiu somente indivíduos saudáveis. Acreditase que isso possa justificar os resultados do presente estudo, uma vez que um dos critérios 
do diagnóstico de demência é uma perda da capacidade funcional do indivíduo. ${ }^{24}$

Outro achado deste trabalho é uma associação entre memória episódica e três atividades instrumentais de vida diária. Esta questão passa a ser relevante, quando se considera que as atividades instrumentais estão mais interligadas às funções corticais superiores do que as básicas, relacionadas mais ao autocuidado. ${ }^{6,16}$ Seria esperada, portanto, maior associação com a memória episódica das primeiras do que das segundas. No entanto, não foi encontrado nenhum estudo que abordasse tal relação sob esta perspectiva. Além disso, os achados não são capazes de dizer se a memória episódica está relacionada às atividades instrumentais de vida diária, de um modo geral, mas evidenciam que ela está interligada mais a alguns itens que a outros.

Cabe reforçar que os estudos sobre funcionalidade e memória episódica no processo de envelhecimento apresentam muitas diferenças relativas aos aspectos metodológicos e ao emprego de instrumentos que avaliam tanto a capacidade funcional quanto a memória episódica, podendo servir de justificativa para as divergências dos resultados entre eles.

Algumas limitações deste estudo merecem ser citadas. Primeiro, optou-se, como critério de exclusão, pelo diagnóstico clínico de quadros demenciais e de depressão, e não uma avaliação por instrumentos de triagem. Dessa forma, o controle de tais critérios ficou a cargo da sensibilidade diagnóstica, sendo que alguns indivíduos podem ter sido incluídos, mesmo tendo algum comprometimento cognitivo leve. Outro fator que merece atenção foi a seleção da amostra, realizada, em quase sua totalidade, em centros de convivências (somente pouco mais de 18\% foram recrutados na comunidade), cujos idosos tendem a apresentar perfil mais ativo e engajado socialmente, podendo não ser uma representação fidedigna da população idosa brasileira. Além disso, o teste de memória aplicado exige que o indivíduo seja ao menos alfabetizado, o que obriga a não incluir os analfabetos e faz com que esta amostra não seja representativa dessa parcela.

\section{CONCLUSÕES}

De maneira geral, o presente estudo não encontrou correlação entre variáveis sociodemográficas e capacidade funcional, nem desta com o desempenho em tarefa de memória episódica, quando a primeira foi analisada utilizando escores totais. No entanto, foi encontrada associação entre memória episódica e três atividades instrumentais de vida diária (arrumar a casa, fazer compras e sair de casa sozinho usando um transporte público não adaptado), que é inconclusiva quanto à participação da memória episódica na capacidade funcional e vice-versa. Além disso, este estudo revelou também associação com significância estatística entre memória episódica e escolaridade.

Acredita-se que estes resultados possam ser justificados pelo fato de a amostra ser constituída, em sua maioria, por idosos de centros de convivência, que são em geral mais ativos e estimulados física e cognitivamente. A hipótese de que no envelhecimento saudável existe a capacidade de, frente às perdas graduais na memória episódica, compensá-las utilizando outras estratégias e recursos, deve receber maior atenção.

E ainda, estudos longitudinais, em que se permite verificar relações de causalidade, com instrumentos padronizados para que se possa fazer um confronto direto de resultados, amostras maiores e mais heterogêneas e com a introdução da variável autoeficácia e sua possível relação com a capacidade funcional, devem ser o foco de futuras pesquisas sobre o tema. 


\section{REFERÊNCIAS}

1. Almeida OP. Queixa de problemas com memória e o diagnóstico de demência. Arq Neuropsiquiatr 1998;56(3):412-18.

2. Kinm S, Giovanello KS. The effects of attention on age-related relational memory deficits: evidence from a novel attentional manipulation. Psychol Aging 2011;26(3):678-88.

3. Salthouse TA. Theoretical perspectives on cognitive aging. Hillsdale: Lawrence Erlbaum Assciates; 1991.

4. Yassuda MS, Lasca VB, Neri AL. Meta-memória e auto-eficácia: um estudo de validação de instrumentos de pesquisa sobre memória e envelhecimento. Psicol Reflex Crit 2005;18(1):78-9.

5. Yassuda MS, Batistoni SST, Fortes AG, Neri AL. Treino de memória no idoso saudável: Benefícios e Mecanismos. Psicol Reflex Criti 2006; 19(3):470-81.

6. Bear MF, Connors BW, Paradiso MA. Sistemas de Memória. In: Bear MF, Connors BW, Paradiso MA. Neurociência: desvendando o sistema nervoso. $3^{\mathrm{a}} \mathrm{ed}$. Porto Alegre: Artmed; 2008. p. 739-74.

7. Guimarães LHCT, Galdino DCA, Martins FLM, Abreu SR, Lima M, Vitorino DFM. Avaliação da capacidade funcional de idosos em tratamento fisioterapêutico. Rev Neurociênc 2004;12(3):130-3.

8. Brasil. Ministério da Saúde, Secretaria de Atenção à Saúde, Departamento de Atenção à Saúde. Envelhecimento e saúde da pessoa idosa. Brasília, DF: Ministério da Saúde; 2006. (Cadernos de Atenção Básica no 19). (Série A. Normas e Manuais Técnicos).

9. Alves LC, Leite IC, Machado CJ. Fatores associados à incapacidade funcional dos idosos no Brasil: análise multinível. Rev Saúde Pública 2010;44(3):1-11.

10. Fiedler MM, Peres KG. Capacidade funcional e fatores associados em idosos do sul do Brasil: um estudo de base populacional. Cad Saúde Pública 2008;24(2):409-15.

11. Giacomin KC, Peixoto SV, Uchoa E,Lima-Costa MF. Estudo de base populacional dos fatores associados à incapacidade funcional entre idosos na Região Metropolitana de Belo Horizonte, Minas Gerais, Brasil. Cad Saúde Pública 2008;24(6):1260-70.

12. Santos KA, Koszuoski K, Dias-da-Costa JS, Pattussi MP. Fatores associados com a incapacidade funcional em idosos do município de Guatambu, Santa Catarina, Brasil. Cad Saúde Pública 2007;23(11):2781-8.
13. Parahiba MI, Simões CCS. A prevalência de incapacidade funcional em idosos no Brasil. Ciênc Saúde Coletiva 2006;11(4):967-74.

14. Maciel ACC, Guerra RO. Fatores associados à alteração da mobilidade em idosos residentes na comunidade. Rev Bras Fisioter 2005;9(1):19-23.

15. Conversor MER, Lartelli I. Caracterização e análise do estado mental e funcional de idosos institucionalizados em instituições públicas de longa permanência. J Bras Psiquiatr 2007;56(4):267-72.

16. Maciel ACC, Guerra RO. Influência dos fatores biopsicossociais sobre a capacidade funcional de idosos residentes no nordeste do Brasil. Rev Bras Epidemiol 2007;10(2):179-89.

17. Siqueira AB, Cordeiro RC, Perracini MR, Ramos LB. Impacto funcional da internação hospitalar de pacientes idosos. Rev Saúde Pública 2004; 38(5):687-94.

18. Lino VTS, Pereira SRM, Camacho LAB, Ribeiro Filho ST, Buksman S. Adaptação transcultural da escala de independência em atividades de vida diária (Escala de Katz). Cad Saúde Pública 2008;24(1):103-12.

19. Paixão-Junior CM, Reichenrein ME. Uma revisão sobre instrumentos de avaliação do estado funcional do idoso. Cad Saúde Pública 2005;21(1):7-19.

20. Ramos LR, Simoes EJ, Albert MS. Dependence in activities of daily living and cognitive impairment strongly predicted mortality in older urban residents in Brazil: a 2-year follow-up. J Am Geriatr Soc 2001;49(9):1168-75.

21. Paulo DLV, Yassuda MS. Queixa de memória de idosos e sua relação com escolaridade, desempenho cognitivo e sintomas de depressão e ansiedade. Rev Psiquiatr Clín 2010;37(1):23-6.

22. Tucker-Drob EM. Neurocognitive functions and everyday functions change together in old age. Neuropsychology 2011;25(3):368-77.

23. Farias ST, Cahn-Weiner DA, Harvey DJ, Reed BR, Mungas D, Kramer JH, et al. Longitudinal changes in memory and executive functioning are associated with longitudinal chance in instrumental activities of Daily Living in older adults. Clin Neuropsychol 2009;23(3):446-61.

24. Cahn-Weiner DA, Farias ST, Julian L, Harvey DJ, Kramer JH, Reed BR, et al. Cognitive and neuroimaging predictors of instrumentals activities de daily living. J Int Neuropsychol Soc 2007;13(5):747-57. 
25. Katz S, Downs TD, Cash HR, Grotz RC. Progress in development of the Index of ADL. Gerontologist 1970;10(1):20-30.

26. Lawton MP, Brody EM. Assessment o folder people: self-maintaining and activities of daily living. Gerontologist 1960;9(3):179-86.

27. West RL, Thorn R M. Goal-setting, self-efficacy, and memory performance in older and younger adults. Exp Aging Res 2001;27(1):41-65.

28. Pergher GK, Stein LM. Compreendendo o esquecimento: teorias clássicas e seus fundamentos experimentais. Psicol USP 2003;14(1):129-55.

29. Instituto Brasileiro de Geografia e Estatística. Censo demográfico 2010: características gerais da população, religião e pessoas com deficiência [Internet]. Brasília: Instituto Brasileiro de Geografia e Estatística; [acesso em 07 jun 2013]. Disponível em: ftp://ftp.ibge.gov.br/ Censos/Censo_Demografico_2010/Caracteristicas_ Gerais_Religiao_Deficiencia/tab1_1.pdf.
30. Salami A, Eriksson E, Nyberg L. Opposing effects of aging on large-scale brain system for memory encoding and cognitive control. J Neurosci 2012;32(31):10749-57.

31. St-Laurent M, Abdi H, Burianová H, Grady CL. Influence of aging on the neural correlates of autobiographical, episodic and semantic memory retrieval. J Cogn Neurosci 2011;23(12):4150-63.

32. Zahodne LB, Glymour MM, Sparks C, Bontempo D, Dixon RA, MacDonald SWS, et al. Education does not slow cognitive decline with aging: 12-year evidence from the Victoria longitudinal study. J Int Neuropsychol Soc 2011;17(6):1039-46.

33. Small BJ, Dixon RA, McArdle JJ. Tracking cognition: healthy changes from 55 to 95 years of age. J Gerontol Ser B, Psychol Sci Soc Sci 2011;66B(Supp 1):i153-63.

34. Lachman ME, Agrigoroaei S, Murphy C, Tun PA. Frequent cognitive activity compensates for education differences in episodic memory. Am J Geriatr Psychiatry 2010;18(1):4-10. 